\title{
First-Principles Calculations of a New Semi-Conductive Carbon Allotrope Named ABF-Carbon
}

\author{
Felipe L. Oliveira ${ }^{\circledR a}$ and Pierre M. Esteves ${ }^{\circledR} * a$ \\ ${ }^{a}$ Instituto de Química, Universidade Federal do Rio de Janeiro, Av. Athos da Silveira Ramos, 149, \\ CT, Bl. A-622, Cidade Universitária, Ilha do Fundão, 21941-909 Rio de Janeiro-RJ, Brazil
}

\begin{abstract}
Carbon is an extremely versatile element and can generate a plethora of structures with distinct properties. Proposing new possible metastable allotropic structures for carbon has been the subject of increasing interest over the past few years. In this contribution, we present a new carbon allotrope, named ABF-Carbon, based on the connection of spiropentadiene molecular motif and $\mathrm{sp}^{3}$ carbon atoms. This new structure is a metastable carbon allotrope formed by 6 carbon atoms, being $2 \mathrm{sp}^{2}$ and $4 \mathrm{sp}^{3}$, in a body-centered tetragonal with space group $I \overline{4} m 2$ (space group 119) and point group $D_{2 d}^{11}$. By first-principles calculations using the density functional theory (DFT), we predict that this new structure is mechanically and structurally stable and presents thermal stability up to $900 \mathrm{~K}$. The calculations also show that ABF-Carbon presents a direct bandgap of $2.39 \mathrm{eV}$ and higher cohesive energy than other carbon allotropes, such as T-Carbon and Y-Carbon/1-diamondyne.
\end{abstract}

Keywords: allotropes, carbon, carbon allotrope, semi-conductive carbon

\section{Introduction}

Carbon is one of the most versatile elements of the periodic table. Due to its ability to present itself in different hybridizations several distinct structures, which are denominated allotropes, ${ }^{1}$ can be conceived using only carbon atoms. In addition to its well-known natural allotropic forms: graphite, diamond, and lonsdaleite, a wide variety of new structures have been synthesized in recent decades. The most remarkable examples include graphene, ${ }^{2}$ fullerenes, ${ }^{3}$ nanotubes, ${ }^{4}$ carbyne, ${ }^{5,6}$ graphdiyne, ${ }^{7}$ T-Carbon, ${ }^{8,9}$ cyclo[18]carbon, ${ }^{10,11}$ and many other nanoallotropes. ${ }^{12}$

Besides those structures, several other allotropic forms such as Y-Carbon, ${ }^{13}$ hexagonal carbon foams, ${ }^{14}$ biphenylene carbon, ${ }^{15}$ tetragraphenes, ${ }^{16,17} \mathrm{~K}_{6}$ carbon, ${ }^{18}$ C $3,{ }^{19}$ schwarzites, ${ }^{20}$ Pentagon-Carbon, ${ }^{21}$ Spiro-Carbon, ${ }^{22}$ $n$-diamondynes, ${ }^{23}$ and many others have been theoretically proposed and their properties computationally studied. Most of these structures are compiled in the Samara Carbon Allotrope database (SACADA). ${ }^{24}$

The successful experimental observation of T-Carbon, ${ }^{8,9}$ after its theoretical proposal by Sheng et al..$^{25}$ in 2011 and the recent synthesis of cyclo[18]carbon, ${ }^{10,11}$ following a slight modification of the method proposed in 1991 by Rubin et al.,,$^{26,27}$ brought vigorous attention to carbon

\footnotetext{
*e-mail: pesteves@iq.ufrj.br
}

technology stimulating the imagination and accelerating the race for the description of new possible allotropic forms of carbon-based on theoretical calculations. ${ }^{28}$

In 2018, we presented ${ }^{23}$ the generalization of the concept of inserting acetylene units among existing allotropes to generate a whole family of new carbon allotropes, called $n$-diamondynes. In the same year, in an entirely independent way, Zhao et al..$^{29}$ used a synthetic route similar to the one proposed in our article to generate a material very similar to one of the members of this family, the 2-diamondyne. Even though the authors were unable to obtain a crystalline material, and therefore it is not precisely the 2-diamondyne, this was an important step towards the synthesis of this allotrope.

In a previous publication, ${ }^{30}$ we showed that it is possible to use the spiropentadiene molecule as a structural motif to build a new carbon allotrope composed of $\mathrm{sp}^{2}$ and $\mathrm{sp}^{3}$ carbon atoms, called Spiro-Carbon, directly connecting the spiro units throughout double bonds..$^{22}$ This direct connection between the molecular motifs allows that the double bonds of the spiro unit can form a conjugated system, similar to infinite polyethylene, thus generating a metallic structure. On the other hand, it is also possible to connect the spiropentadiene motif directly to $\mathrm{sp}^{3}$ carbon atoms, as illustrated in Figure 1, forming a structure with ABF topology that resembles Spiro-Carbon but do not present the conjugation between double bonds, thus 


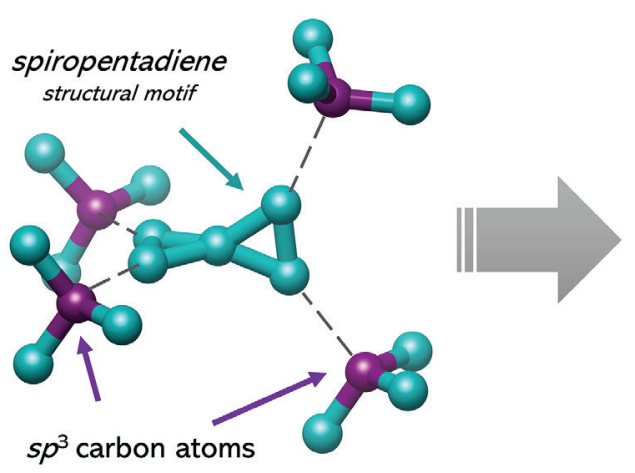

Figure 1. Simplified scheme of the building process for ABF-Carbon structure.

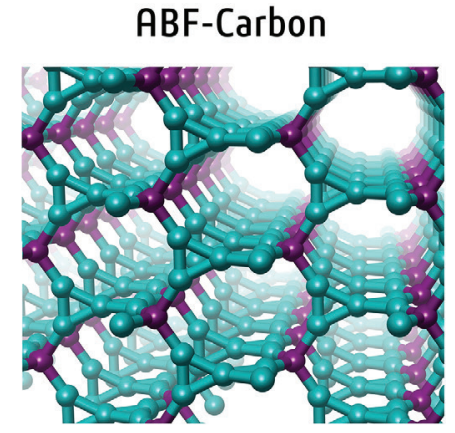

generating a potential new 3D semi-conductor carbon allotrope.

In the present work, we explore the structural and electronic characteristics of this new hypothetical structure, named ABF-Carbon, showing that this new structure is dynamically and thermally stable and presents higher cohesive energy than other carbon allotropes. The results also confirm the semi-conductive character of the structure, showing that it is possible to use a single structural motif to generate materials with different electronic properties by the precise control of how these motifs are interconnected.

\section{Methodology}

All $a b$ initio calculations were carried out based on density functional theory (DFT) calculations under periodic boundary conditions approach as implemented in the PWscf code of Quantum ESPRESSO version 6.4. ${ }^{31,32}$ Exchange and correlation effects are treated with generalized gradient approximation (GGA) of Perdew-Burke-Ernzerhof (PBE) functional ${ }^{33}$ and ultrasoft pseudopotentials ${ }^{34}$ was used to describe the nuclei and core electrons. The Kohn-Sham orbitals are expanded in a plane-wave basis set with a kinetic energy cutoff of 80 Ry and 800 Ry for the charge density for all calculations and the first Brillouin Zone integration was performed in a $(1,1,1)$ shifted $12 \times 12 \times 12$ Monkhorst-Pack ${ }^{35} \mathrm{k}$-point mesh (chosen based on systematically checking the convergence with total energy as presented in Figure S1 of Supplementary Information (SI) section). The dispersion forces were treated with the D3 correction method proposed by Grimme et al. ${ }^{36}$ Atomic positions and cell parameters were simultaneously fully optimized using the Broyden-Fletcher-Goldfarb-Shanno (BFGS) quasi-Newton minimization algorithm ${ }^{37}$ until the Hellmann-Feynman forces acting on the atoms were lower than $10^{-5} \mathrm{Ry} / \mathrm{Bohr}$ and total energy changes less than $10^{-4} \mathrm{Ry}$.

The band diagram, the density of states (DOS), and the projected density of states (pDOS) were calculated in a dense k-points grid of $36 \times 36 \times 36$ using the tetrahedron method $^{38}$ for occupations. The phonon dispersion was calculated based on the density-functional perturbation theory approach, as implemented in the phonon code, ${ }^{39}$ with a threshold for self-consistency of $10^{-16} \mathrm{Ry}$ in a q-mesh of $6 \times 6 \times 6$ and Fourier interpolated along with the points of high symmetry of the Brillouin zone.

Cohesive energy $\left(E_{c}\right)$ was calculated as $E_{c}=E_{\text {carbon }}-E_{\text {tot }} n^{-1}$ where $E_{\text {tot }}$ is the total energy of the unit cell, $E_{\text {carbon }}$ is the energy of an isolated carbon atom on the ground state $\left({ }^{3} \mathrm{P}_{0}\right)$ and $\mathrm{n}$ is the number of atoms in the unit cell. Elastic constants $(\mathrm{C})$ of the stiffness matrix were obtained by calculating the stress $(\sigma)$ resulting from a given strain $(\epsilon)$ and determining the respective elastic constant based on the well-known Hook's law equation $\sigma_{\mathrm{i}}=\sum_{\mathrm{j}=1}^{6} \mathrm{C}_{\mathrm{ij}} \epsilon_{\mathrm{ij}}$ using the stress-strain routines as implemented in the thermo_pw package. The tensorial analysis of the stiffness matrix was performed using the Elate tool..$^{40}$ The Vicker's hardness $\left(\mathrm{H}_{v}\right)$ of the polycrystalline material was calculated based on the semi-empirical model of Chen et al. ${ }^{41}$ given by the expression $\mathrm{H}_{\mathrm{v}}=2\left(\mathrm{k}^{2} \mathrm{G}\right)^{0.585}-3$, where the parameter $\mathrm{k}$ is the Pugh's modulus and is given by the ratio between shear (G) and bulk (B) modulus $(\mathrm{k}=\mathrm{G} / \mathrm{B})$.

The Born-Oppenheimer ab initio molecular dynamics (AIMD) simulation was performed based on periodic boundary conditions (3D PBC) within a $2 \times 2 \times 2$ supercell, using the Quickstep program as implemented in the cp2k package. ${ }^{42,43}$ The Perdew-Burke-Ernzerhof and Grimme's D3 dispersion correction functional (PBE-D3) with Goedecker, Teter, and Hutter $(\mathrm{GTH})^{44,45}$ norm-conserving pseudopotential was used to describe core electrons, and the GTH-valence double-zeta-polarized Gaussian basis combined with a plane-wave basis (PBE-D3/ GPW/DZVP/GTH) set with an energy cutoff of $900 \mathrm{Ry}$ is selected for the AIMD simulations (chosen based on systematically checking the convergence with total energy as presented in Figure S2 of SI section). It is important to highlight that the difference in the cutoff energy between 
QuantumESPRESSO and cp2k is a consequence of the fact that $c p 2 k$ uses a combination of plane and Gaussian waves to represent electronic density, different from QuantumESPRESSO which uses only plane waves. The generalized Langevin equation (GLE) ${ }^{46}$ thermostat for the NVT ensemble and a time step of $0.5 \mathrm{fs}$ were used for all simulations.

\section{Results and Discussion}

The idealized structure of ABF-Carbon possess 12 carbon atoms in a primitive body-centered tetragonal cell with space group $I \overline{4} m 2$ (space group 119) and point group $D_{2 d}^{11}$. The resulting geometry from full variable cell optimization afforded a conventional tetragonal cell with parameters $a=b=3.811 \AA$, $c=8.726 \AA$, angles $\alpha=\beta=\gamma=90^{\circ}$ and density $1.89 \mathrm{~g} \mathrm{~cm}^{-3}$. The topology of this structure can be represented by a 3D network of the ABF type and based on that this structure was named ABF-Carbon. The crystallographic information file containing the atomic coordinates for the primitive cell is provided in the SI section.

The three nonequivalent atoms occupy the Wyckoff sites $2 \mathrm{a}, 2 \mathrm{c}$, and $8 \mathrm{i}$. The $\mathrm{C} 1$ (highlighted in green on Figure 2e) occupies the $2 \mathrm{a}$ site at fractional coordinates $(0.0,0.0,0.0), \mathrm{C} 2$ (yellow) occupies the 8i site at fractional coordinates $(0.32651,0.00000,0.90266)$ and $\mathrm{C} 3$ (blue) occupies the $2 \mathrm{c}$ site at fractional coordinates $(0.00,0.50$, 0.25 ). Regarding the covalent topology it is possible to distinguish three different bond types: (i) the bonds that connect the spiro motifs to the $\mathrm{sp}^{3}$ carbons $(\mathrm{C} 2-\mathrm{C} 3)$ with $1.507 \AA$, a characteristic length of simple bonds, ${ }^{47,48}$ (ii) the bonds of the central $\mathrm{sp}^{3}$ carbon of the spiro unit (C1-C2) with lengths of $1.487 \AA$, analogous to the equivalent in the parent spiropentadiene (1.483 $\AA$, see SI section for more detail) and compatible with single $\mathrm{C}-\mathrm{C}$ bonds and (iii) the bonds between the $\mathrm{sp}^{2}$ carbon atoms $(\mathrm{C} 2=\mathrm{C} 2)$ of the spiro unit with lengths of $1.322 \AA$, close to the equivalent in the parent spiropentadiene $(1.31 \AA)$ and presenting characteristic length of double bonds..$^{48}$

The angles between the two three-member rings of the spiro moiety are $90^{\circ}$ to each other, indicating that there is no distortion of this structural motif in the formation of the extended structure. The internal ring angles are $63.6^{\circ}$ $(\mathrm{C} 2-\mathrm{C} 2-\mathrm{C} 1$ and $\mathrm{C} 1-\mathrm{C} 2-\mathrm{C} 2)$ and $52.8^{\circ}(\mathrm{C} 2-\mathrm{C} 1-\mathrm{C} 2)$. These values are virtually equivalent to those presented by the molecular analog. The angles of the $\mathrm{sp}^{3}$ carbon atom are 108.5 and $111.4^{\circ}$, close to the presented by a regular $\mathrm{sp}^{3}$ carbon atom of $109.5^{\circ}$.

One interesting characteristic is the fact that in ABF-Carbon the bond lengths and angles of the spiro unit presented values very close to their molecular analog, the

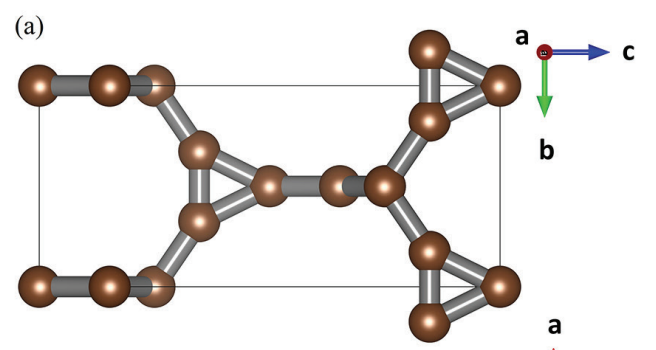

(d)
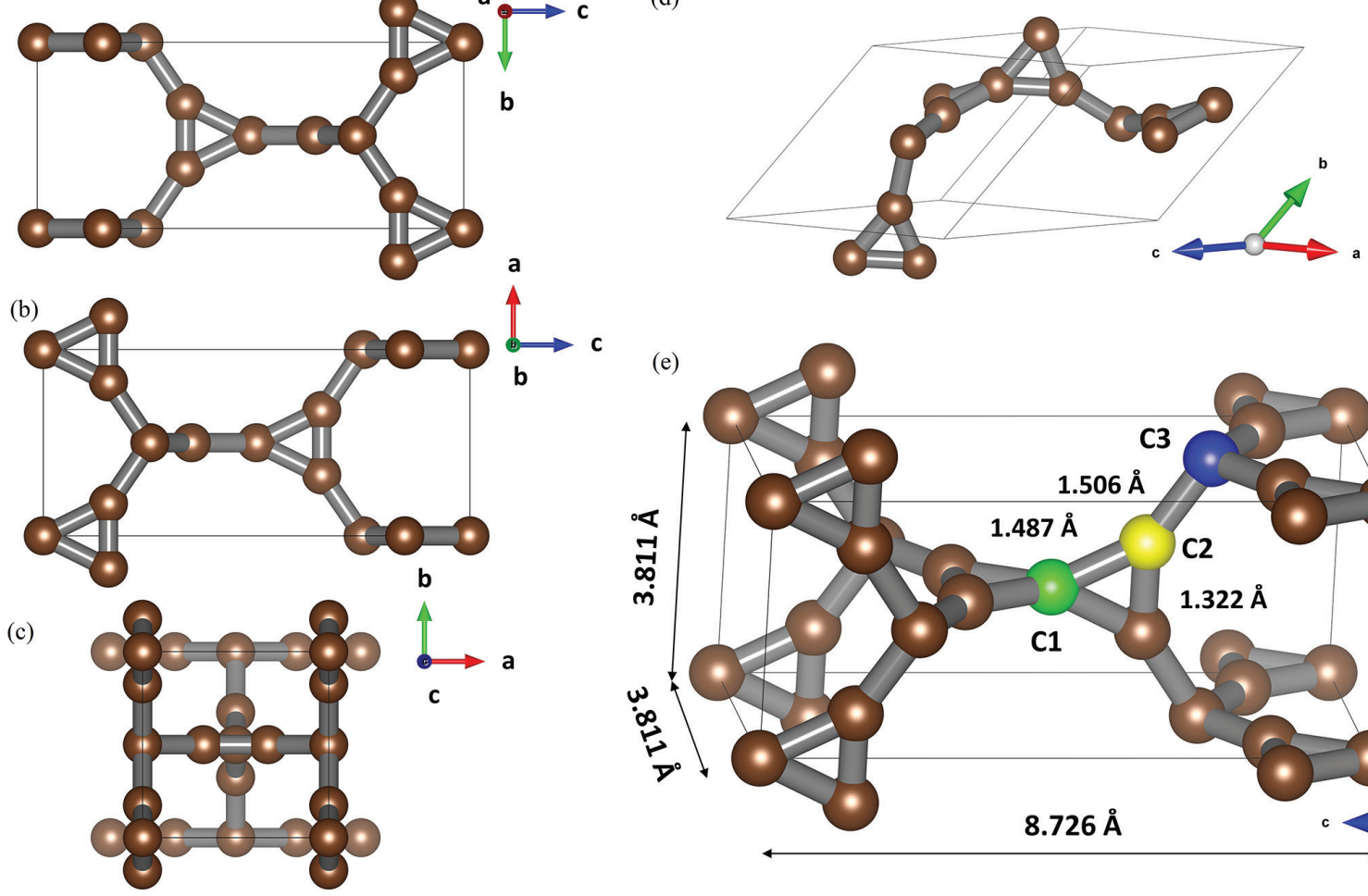

(b)

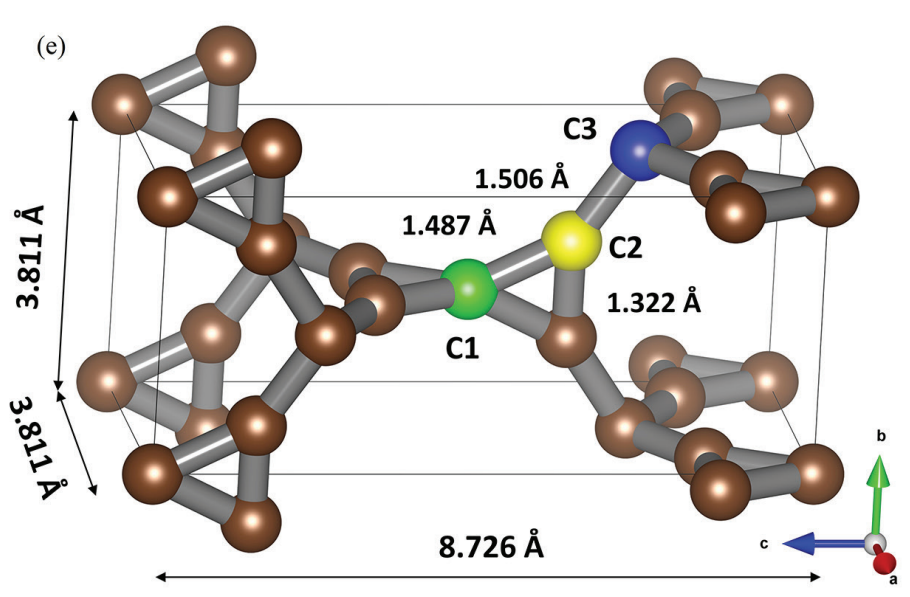

Figure 2. Atomic representation of the tetragonal structure of ABF-Carbon, (a) view along $a$ axis; (b) view along $b$ axis; (c) view along $c$ axis; (d) representation of the primitive unit cell; (e) representation of the tetragonal cell with the three nonequivalent atoms highlighted. 
spiropentadiene, than the equivalent connections and angles in the Spiro-Carbon structure. This is a consequence of the fact that in ABF-Carbon structure the spiro units are directly linked to $\mathrm{sp}^{3}$ carbon atoms which prevent the conjugation between double bonds and causing them to form classical double and single bonds. This allows one to anticipate that this structure will present a semi-conductive character with a relatively large bandgap, due to the lack of conjugation between the $\pi$ orbitals.

To explore the stability of this proposed new structure, three main points were analyzed: (i) vibrational stability based on the phonon dispersion; (ii) thermal stability based on ab initio molecular dynamics (AIMD), and (iii) mechanical stability analysis of the elastic matrix. First, the phonon dispersion spectra were calculated along with the principal high symmetry points of the first Brillouin zone ${ }^{49}$ and are displayed in Figure 3a. No imaginary frequency is observed throughout the phonon dispersion spectra, indicating that ABF-Carbon's structure corresponds to a minimum in the potential energy surface.
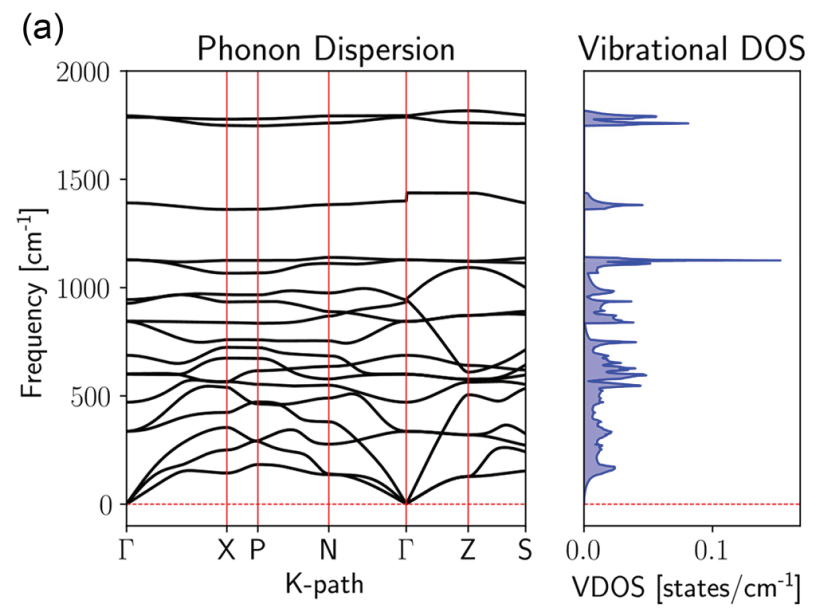

(c)

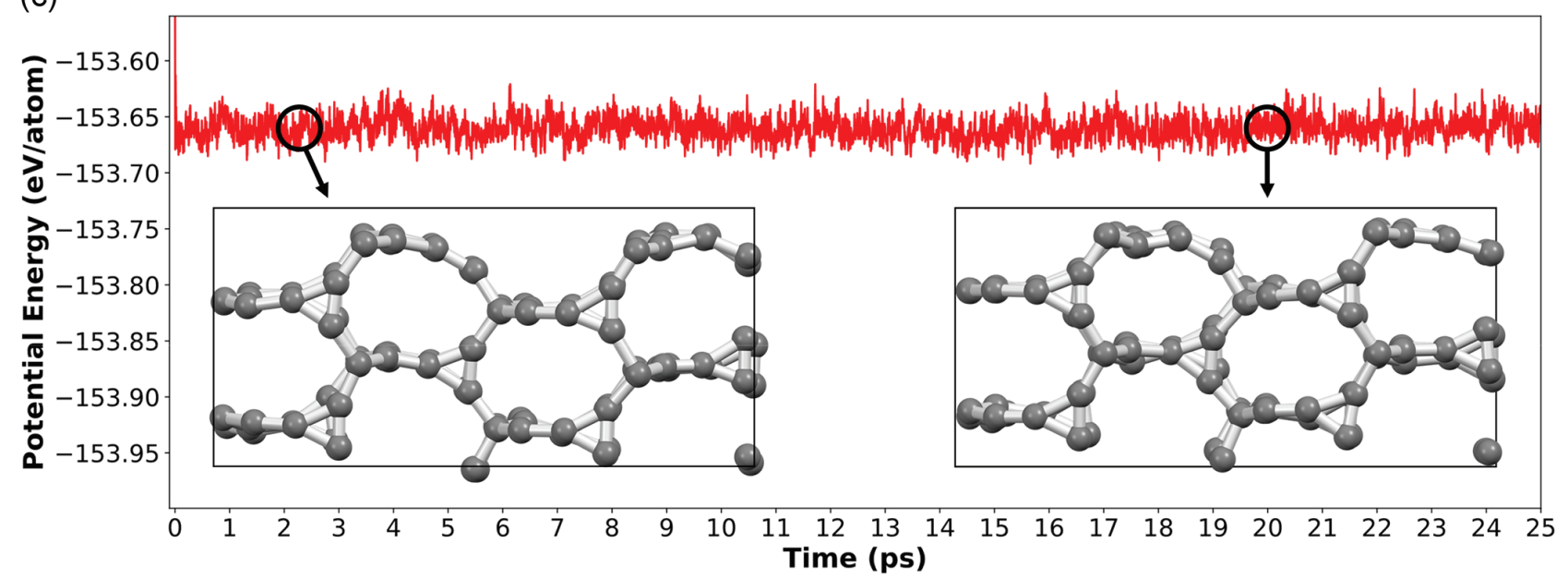

Figure 3. (a) Plot of the phonon distribution along with some high symmetry directions of the first Brillouin zone and the corresponding vibrational density of states (DOS) and the selected k-path throughout the first Brillouin zone (insert); (b) total energy per atom as a function of specific volume for different carbon allotropes; (c) potential energy as a function of simulation time and selected snapshots, at 2 and 20 ps, of Born-Oppenheimer AIMD at $900 \mathrm{~K}$.
To better understand the relative energetic stability of ABF-Carbon, Figure $3 b$ shows the variation of cohesive energy per atom of several carbon allotropes (for atomistically detailed structures see Figure S6, SI section) against its unit cell volume per atom. As expected, ABF-Carbon presented a single minimum and exhibited lower cohesive energy than graphite or diamond, the most stable natural allotropic phases. Even so, ABF-Carbon has cohesive energy equivalent to that presented by carbyne and greater than several other allotropes such as 1-diamondyne/Y-Carbon by about $2.3 \mathrm{kcal} \mathrm{mol}^{-1}(0.1 \mathrm{eV})$ per atom at zero pressure, and T-Carbon, that has already been successfully synthesized, by about $6.1 \mathrm{kcal} \mathrm{mol}^{-1}$ $(0.26 \mathrm{eV})$, as shown in Table 1.

Interestingly, ABF-Carbon presented cohesive energy of about $0.05 \mathrm{eV}$ (1.0 kcal mol$\left.{ }^{-1}\right)$ smaller than Spiro-Carbon. In general, it is expected that a structure presenting more carbon atoms with $\mathrm{sp}^{3}$ hybridization will present greater stability and higher cohesive energy. Nevertheless, even $\mathrm{ABF}-\mathrm{Carbon}$ presenting a $\mathrm{sp}^{3} / \mathrm{sp}^{2}$ ratio of $1 / 2$, which is

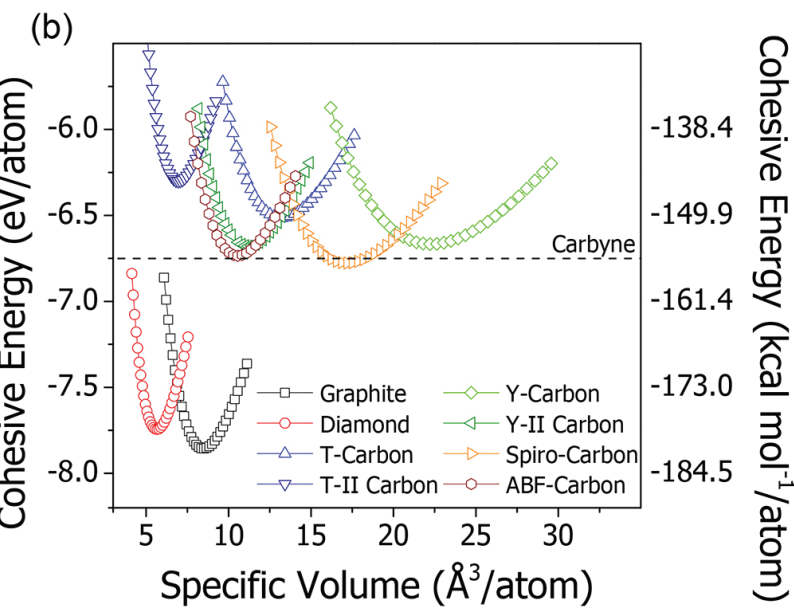


Table 1. Relative and cohesive energy per atom for ABF-Carbon and other carbon allotropes

\begin{tabular}{lcc}
\hline Structure & $\begin{array}{c}\text { Relative energy / } \\
\left(\mathrm{eV}^{-10)^{-1}}\right)\end{array}$ & $\begin{array}{c}\text { Cohesive energy / } \\
\left(\mathrm{eV}^{-1} \text { atom }^{-1}\right)\end{array}$ \\
\hline Graphite & 0.000 & 7.856 \\
Diamond & 0.112 & 7.744 \\
Spiro-Carbon & 1.079 & 6.777 \\
Carbyne & 1.106 & 6.750 \\
ABF-Carbon & 1.121 & 6.735 \\
Y-Carbon & 1.189 & 6.667 \\
Y-II Carbon & 1.177 & 6.679 \\
T-Carbon & 1.342 & 6.514 \\
T-II Carbon & 1.554 & 6.302 \\
\hline
\end{tabular}

higher than the 1/4 ratio presented by Spiro-Carbon, it possesses smaller cohesive energy. This phenomenon is quite unusual and could be related to the fact that graphite has greater cohesive energy than diamond. It is possible that the electronic delocalization as a consequence of the conjugated $\pi$ bonds formed, as in graphite and SpiroCarbon, is a better stabilizing factor than the frontal overlap of the orbitals generated by the formation of $\sigma$ bonds in structures such as the diamond and ABF-Carbon. This phenomenon still does not have a conclusive answer in the literature, and further research must be done to explain this counterintuitive result.

Still on the relative stability of the new structure, we estimate the heat of formation from an isodesmic reaction, ${ }^{50}$ i.e., a hypothetical reaction in which the number and type of chemical bonds are conserved. Using the hypothetical reaction spiropentadiene $\left(\mathrm{C}_{5} \mathrm{H}_{4}\right)+$ diamond $\left(\mathrm{C}_{2}\right) \rightarrow$ ABF-Carbon $\left(\mathrm{C}_{6}\right)+$ methane $\left(\mathrm{CH}_{4}\right)$ the heat of formation is $-17.1 \mathrm{kcal} \mathrm{mol}^{-1}$, an intermediate value between the formation heats obtained for other carbon allotropes such as T-Carbon $\left(-37.2 \mathrm{kcal} \mathrm{mol}^{-1}\right)$ and Y-Carbon $\left(-12.7 \mathrm{kcal} \mathrm{mol}^{-1}\right)$. For more details, see the SI section.

Next, to investigate the thermal stability of ABF-Carbon Born-Oppenheimer $a b$ initio molecular dynamics (AIMD) simulations were performed under the temperatures of 300 , 600,900 and $1500 \mathrm{~K}$ using a $2 \times 2 \times 2$ supercell (containing 96 atoms) based on the conventional tetragonal cell, with the results being presented in Figure 3c. The system was followed by $25.0 \mathrm{ps}$ of simulation with a time step of $0.5 \mathrm{fs}$, which considering the time relative to the vibrational mode with the highest frequency $\left(1793.04 \mathrm{~cm}^{-1}=18.6 \mathrm{fs}\right)$ is sufficient to calculate 16 points between each vibration. At $900 \mathrm{~K}$ the covalent bond topology remained the same as the optimized structure and only small distortions can be observed in the structure, as illustrated by the selected snapshots presented in Figure 3c, which indicates that ABF-Carbon could remain stable in temperatures as high as $900 \mathrm{~K}$. After 4 ps of simulation at $1500 \mathrm{~K}$ several chemical bonds were broken (Figure S5, SI section), indicating that the degradation temperature of ABF-Carbon is somewhere between 900 and $1500 \mathrm{~K}$.

Finally, regarding ABF-Carbon's mechanical stability the six independent elastic constants $\left(\mathrm{C}_{\mathrm{ij}}\right.$, stiffness in GPa) were calculated based on the stress-strain relations, and the Voigt-Reuss-Hill average mechanical properties of the polycrystalline material are exhibited in Table 2 compared with the calculated values for diamond and T-Carbon.

Table 2. Elastic constants $\left(\mathrm{C}_{\mathrm{ij}}\right)$, bulk $(\mathrm{B})$, shear $(\mathrm{G})$, and Young modulus (E), Poisson ratio $(v)$, and density $(\rho)$ calculated for the diamond, T-Carbon, and ABF-Carbon

\begin{tabular}{lccc}
\hline & Diamond & T-Carbon & ABF-Carbon \\
\hline $\mathrm{C}_{11} / \mathrm{GPa}$ & 1106.43 & 202.22 & 481.72 \\
$\mathrm{C}_{33} / \mathrm{GPa}$ & 1106.43 & 202.22 & 560.51 \\
$\mathrm{C}_{44} / \mathrm{GPa}$ & 591.34 & 63.52 & 120.03 \\
$\mathrm{C}_{66} / \mathrm{GPa}$ & 591.34 & 63.52 & 38.09 \\
$\mathrm{C}_{12} / \mathrm{GPa}$ & 153.08 & 165.64 & 10.04 \\
$\mathrm{C}_{13} / \mathrm{GPa}$ & 153.08 & 165.64 & 90.13 \\
$\mathrm{~B} / \mathrm{GPa}$ & 470.86 & 177.80 & 209.37 \\
$\mathrm{G} / \mathrm{GPa}$ & 542.45 & 38.64 & 120.30 \\
$\mathrm{E} / \mathrm{GPa}$ & 1175.83 & 107.88 & 301.40 \\
$\nu /\left(\mathrm{g} \mathrm{cm}^{-3}\right)$ & 0.084 & 0.396 & 0.253 \\
$\rho /\left(\mathrm{g} \mathrm{cm}^{-3}\right)$ & 3.52 & 1.51 & 1.89 \\
\hline
\end{tabular}

Based on Born stability criteria the four necessary and sufficient conditions that must be satisfied to ensure the mechanical stability for a tetragonal lattice are $\mathrm{C}_{11}>\left|\mathrm{C}_{12}\right|$, $2 \mathrm{C}_{13}<\mathrm{C}_{33}\left(\mathrm{C}_{11}+\mathrm{C}_{12}\right), \mathrm{C}_{44}>0$, and $\mathrm{C}_{66}>0 .{ }^{51}$ All these criteria are completely satisfied with ABF-Carbon, therefore it can be concluded that this new proposed structure is mechanically stable.

To further characterize the mechanical behavior on the elastic regime, a complete tensorial analysis of the elastic matrix was conducted and the spatial dependence on planes $x y, x z$, and $y z$ for Young (E) and shear modulus $(\mathrm{G})$, linear compressibility $(\beta)$ and Poisson's ratio $(v)$ are presented in Figure 4. ABF-Carbon presented a significant anisotropy of Young's and shear modulus, especially along the $x y$ plane, a common characteristic in soft and porous materials.

The calculated Vicker's hardness of the polycrystalline material for ABF-Carbon was $14.2 \mathrm{GPa}$, small when compared with diamond $(90.9 \mathrm{GPa}$, in accordance with the experimental value of $96 \pm 5)^{52}$ but close to the calculated 


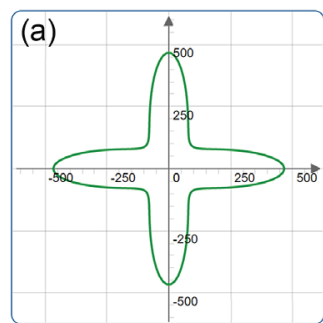

(xy) plane

\section{Young's modulus}

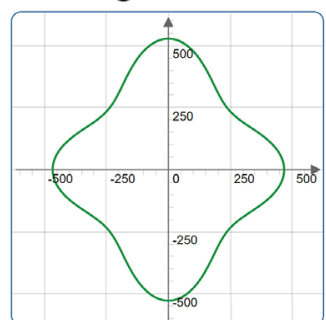

( $x z$ ) plane

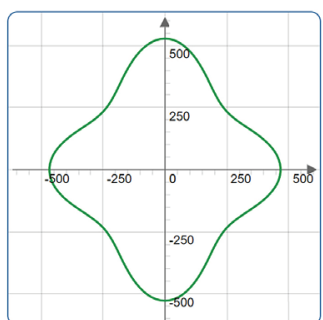

(yz) plane

Linear compressibility

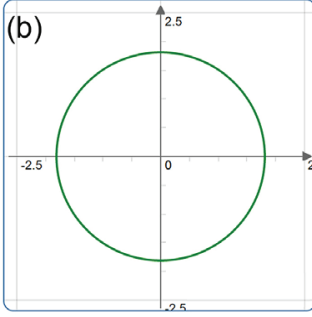

(xy) plane

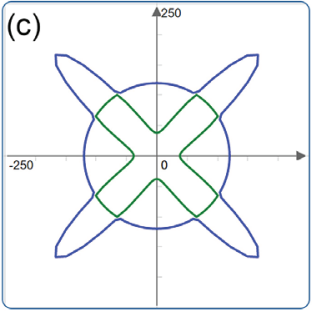

(xy) plane

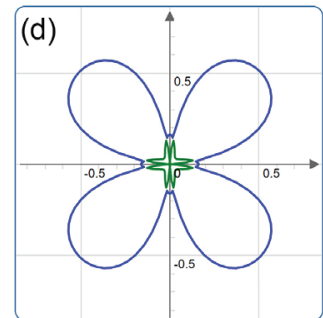

(xy) plane

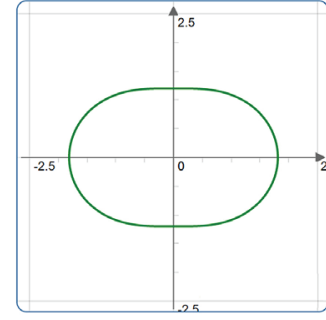

(xz) plane

Shear modulus

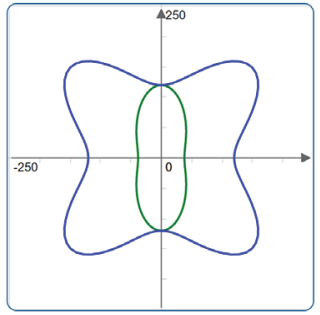

(xz) plane

Poisson's ratio

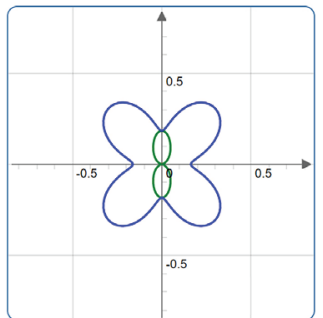

(xz) plane

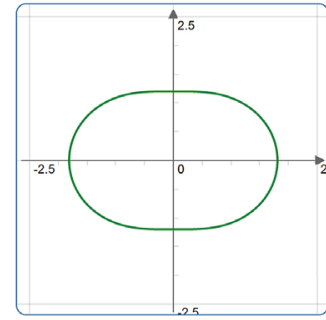

(yz) plane

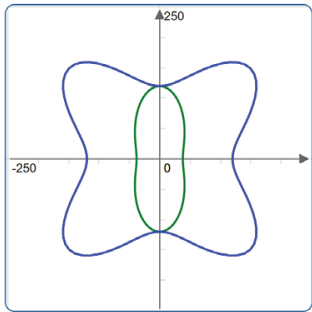

(yz) plane

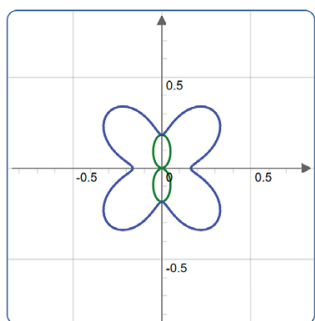

(yz) plane

Figure 4. Spatial dependence of (a) Young's modulus; (b) linear compressibility; (c) shear modulus and (d) Poisson's ratio.

for several other 3D carbon allotropes. Based on the Push's classification of brittleness and ductless, which correlates a high $\mathrm{B} / \mathrm{G}$ ratio to a high ductile material, $\mathrm{ABF}-\mathrm{Carbon}$ might be more ductile than diamond once synthesized.

Now, turning to the electronic properties, the structure of ABF-Carbon, unlike Spiro-Carbon, possess localized double bonds inside the spiro units forming an endoannular bond without the possibility of conjugation, which indicates that this new material may have a semi-conductive character. To explore the electronic characteristics of ABF-Carbon the band diagram calculated at the PBE level is presented in Figure 5a. It is possible to observe a direct bandgap of $1.35 \mathrm{eV}$ at the $\mathrm{X}$ point, confirming the semi-conductive character of this structure. An interesting feature of this structure is the quasi-flat character of the valence band on the $\Gamma-\mathrm{X}$ path of the first Brillouin zone, generating a high electron density of states (ca. 1.8 states $\mathrm{eV}^{-1}$ ) near to the Fermi level formed mainly by the $2 \mathrm{p}$ orbitals $\left(2 \mathrm{p}_{\mathrm{x}}, 2 \mathrm{p}_{\mathrm{y}}\right.$, and $2 p_{z}$ ), a reminiscent behavior of the isolated double bonds $(\mathrm{C} 2=\mathrm{C} 2)$ of the spiro molecular motif, as can be seen in the atom resolved projected density of states (Figure $5 \mathrm{~b}$ ). 
(a)

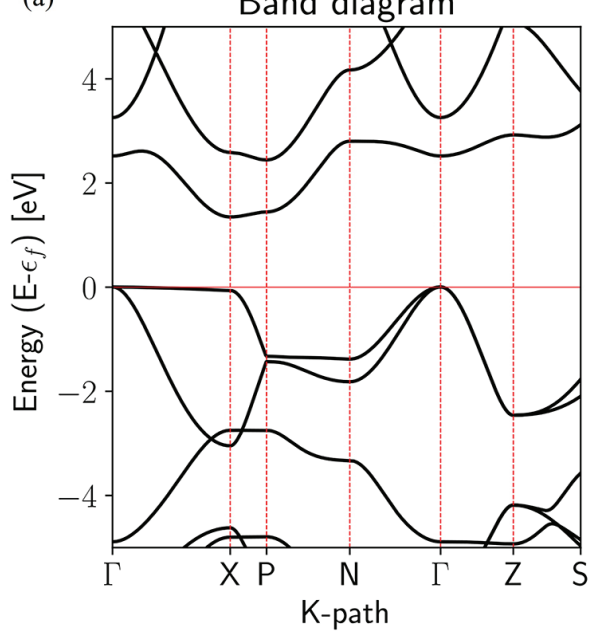

pDOS dist.

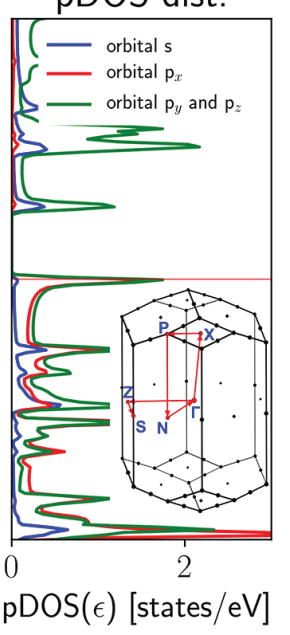

(b)

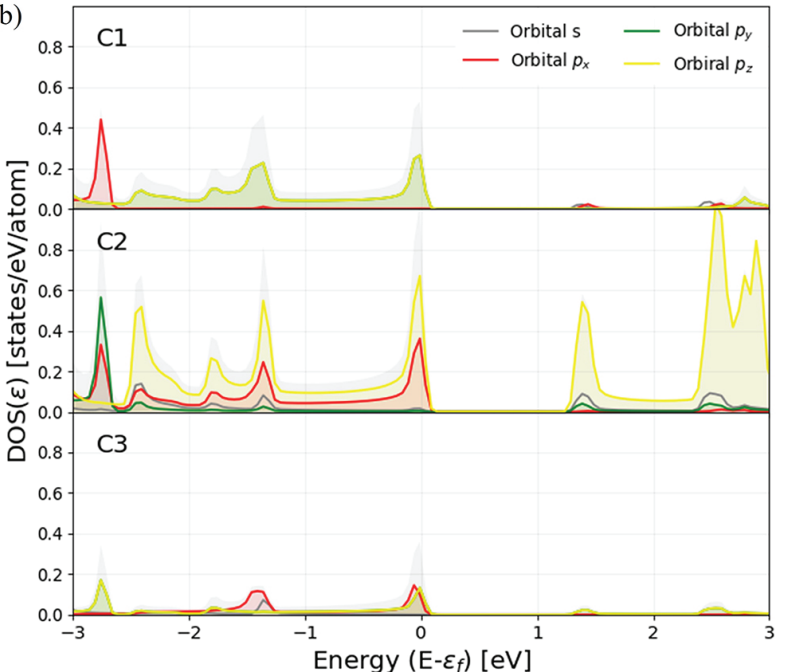

Figure 5. (a) Electronic band dispersion curves along with some high symmetry directions of the Brillouin zone (left), the density of states (right), and the first Brillouin zone (insert) for ABF-Carbon. (b) Atom-resolved projected density of states.

Since the PBE functional is well known to underestimate the bandgap of semiconductors, this property was also calculated using the HSE06 functional presenting the value of $2.39 \mathrm{eV}$. This value is considerably smaller than the calculated for the diamond $(5.39 \mathrm{eV})$, T-Carbon $(3.34 \mathrm{eV})$, and polyynic carbyne $(2.7 \mathrm{eV})$ in the same level of theory, indicating that ABF-Carbon may find interesting applications in semiconductor and optoelectronic devices.

To assist the future characterization of possible synthetic candidates Raman spectra and X-ray diffraction pattern for ABF-Carbon are presented in Figure 6, compared with some other carbon allotropes. The Raman spectrum presented in Figure 6a has a main peak at $1776 \mathrm{~cm}^{-1}$ due to the vibrational mode with symmetry $A_{1}$ and three minor peaks at 1377 , 938 , and $621 \mathrm{~cm}^{-1}$ referent to three $\mathrm{E}$ modes. The X-ray diffraction spectrum, shown in Figure 6b calculated for a wavelength of $1.54059 \AA$, presents the main peak at $25.5^{\circ}$ referring to the Bragg plane (101) and minor peaks in 20.3 and $33.2^{\circ}$, referring to plans (002) and (110), respectively. The calculated ${ }^{13} \mathrm{C}$ NMR chemical shifts presented three different values: $42.2 \mathrm{ppm}$ for the $\mathrm{C} 1$ atom, $128.5 \mathrm{ppm}$ for the $\mathrm{C} 2$ atom, and 39.2 for the $\mathrm{C} 3$ atom, compatible with the expected for the hybridization of these atoms.

\section{Conclusions}

In summary, using first-principles calculations based on DFT we introduce the ABF-Carbon, which to our knowledge is an unreported carbon allotrope, composed of carbon atoms with $\mathrm{sp}^{2}$ and $\mathrm{sp}^{3}$ hybridizations. The results
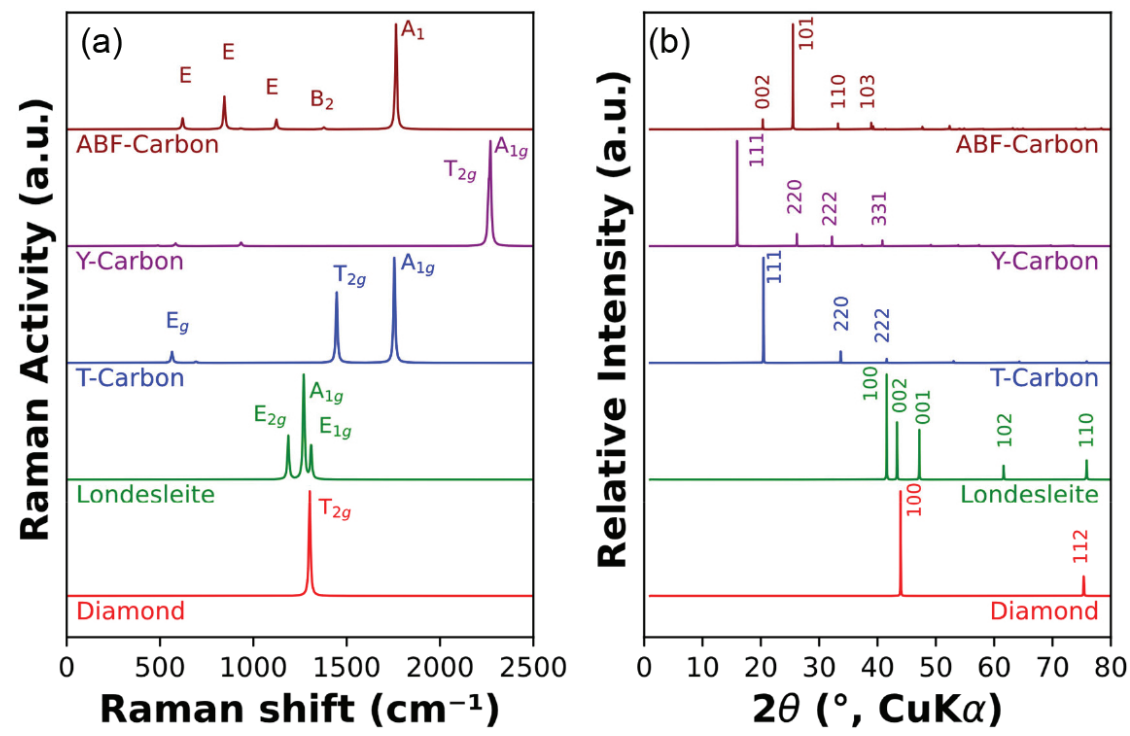

Figure 6. Calculated spectra of (a) Raman shifts and (b) powder X-ray diffraction for ABF-Carbon and several other carbon allotropes. 
show that ABF-Carbon is dynamically, mechanically, and thermally stable up to $900 \mathrm{~K}$. The calculations indicate that ABF-Carbon presents a direct bandgap of $2.39 \mathrm{eV}$ and higher cohesive energy than other carbon allotropes such as T-Carbon and Y-Carbon/1-diamondyne. We also provided calculations of fairly common characterizations such as XRD and Raman spectra in the hope that they will be useful in assisting the characterization of possible synthetic candidates.

\section{Supplementary Information}

Supplementary information (convergence tests, calculations for spiropentadiene, representation of the highsymmetry path on the first Brillouin zone, and fractional coordinates of ABF-carbon) is available free of charge at http://jbcs.sbq.org.br as PDF file.

\section{Acknowledgments}

We acknowledge financial support from CAPES (project 001), CNPq and FAPERJ. The authors would like to thank the Núcleo Avançado de Computação de Alto Desempenho (NACAD) of COPPE/UFRJ for the computational facility.

\section{References}

1. McNaught, A. D.; Wilkinson, A. In IUPAC Compendium of Chemical Terminology; Nič, M.; Jirát, J.; Košata, B.; Jenkins, A.; McNaught, A., eds.; IUPAC: Research Triangle Park, NC, 2009.

2. Novoselov, K. S.; Geim, A. K.; Morozov, S. V.; Jiang, D.; Zhang, Y.; Dubonos, S. V.; Grigorieva, I. V.; Firsov, A. A.; Science 2004, 306, 666.

3. Kroto, H. W.; Heath, J. R.; O’Brien, S. C.; Curl, R. F.; Smalley, R. E.; Nature 1985, 318, 162.

4. Iijima, S.; Nature 1991, 354, 56.

5. Chalifoux, W. A.; Tykwinski, R. R.; Nat. Chem. 2010, 2, 967.

6. Pan, B.; Xiao, J.; Li, J.; Liu, P.; Wang, C.; Yang, G.; Sci. Adv. 2015, 1 , e1500857.

7. Li, G.; Li, Y.; Liu, H.; Guo, Y.; Li, Y.; Zhu, D.; Chem. Commun. 2010, 46, 3256

8. Zhang, J.; Wang, R.; Zhu, X.; Pan, A.; Han, C.; Li, X.; Dan Zhao; Ma, C.; Wang, W.; Su, H.; Niu, C.; Nat. Commun. 2017, 8,683 .

9. Xu, K.; Liu, H.; Shi, Y.-C.; You, J.-Y.; Ma, X.-Y.; Cui, H.-J.; Yan, Q.-B.; Chen, G.-C.; Su, G.; Carbon 2020, 157, 270.

10. Kaiser, K.; Scriven, L. M.; Schulz, F.; Gawel, P.; Gross, L.; Anderson, H. L.; Science 2019, 365, 1299.

11. Scriven, L. M.; Kaiser, K.; Schulz, F.; Sterling, A. J.; Woltering,
S. L.; Gawel, P.; Christensen, K. E.; Anderson, H. L.; Gross, L.; J. Am. Chem. Soc. 2020, 142, 12921.

12. Georgakilas, V.; Perman, J. A.; Tucek, J.; Zboril, R.; Chem. Rev. 2015, 115, 4744.

13. Jo, J. Y.; Kim, B. G.; Phys. Rev. B 2012, 86, 075151.

14. Kuc, A.; Seifert, G.; Phys. Rev. B 2006, 74, 214104.

15. Brunetto, G.; Autreto, P. A. S.; Machado, L. D.; Santos, B. I.; dos Santos, R. P. B.; Galvão, D. S.; J. Phys. Chem. C 2012, 116, 12810 .

16. de Vasconcelos, F. M.; Souza Filho, A. G.; Meunier, V.; Girão, E. C.; Phys. Rev. Mater. 2019, 3, 066002.

17. de Vasconcelos, F. M.; Souza Filho, A. G.; Meunier, V.; Girão, E. C.; Carbon 2020, 167, 403.

18. Niu, C.-Y.; Wang, X.-Q.; Wang, J.-T.; J. Chem. Phys. 2014, $140,054514$.

19. Wang, J.-T.; Chen, C.; Kawazoe, Y.; Phys. Rev. B 2012, 85, 214104.

20. Felix, L. C.; Woellner, C. F.; Galvao, D. S.; Carbon 2020, 157, 670.

21. Zhong, C.; Chen, Y.; Yu, Z.-M.; Xie, Y.; Wang, H.; Yang, S. A.; Zhang, S.; Nat. Commun. 2017, 8, 15641.

22. Oliveira, F. L.; Capaz, R. B.; Esteves, P. M.; ChemPhysChem 2020, 21, 59 .

23. Costa, D. G.; Henrique, F. J. F. S.; Oliveira, F. L.; Capaz, R. B.; Esteves, P. M.; Carbon 2018, 136, 337.

24. Hoffmann, R.; Kabanov, A. A.; Golov, A. A.; Proserpio, D. M.; Angew. Chem., Int. Ed. 2016, 55, 10962.

25. Sheng, X.-L.; Yan, Q.-B.; Ye, F.; Zheng, Q.-R.; Su, G.; Phys. Rev. Lett. 2011, 106, 155703.

26. Rubin, Y.; Kahr, M.; Knobler, C. B.; Diederich, F.; Wilkins, C. L.; J. Am. Chem. Soc. 1991, 113, 495.

27. Rubin, Y.; Knobler, C. B.; Diederich, F.; J. Am. Chem. Soc. 1990, 112, 4966.

28. Hirsch, A.; Nat. Mater. 2010, 9, 868.

29. Zhao, Z.; Das, S.; Xing, G.; Fayon, P.; Heasman, P.; Jay, M.; Bailey, S.; Lambert, C.; Yamada, H.; Wakihara, T.; Trewin, A.; Ben, T.; Qiu, S.; Valtchev, V.; Angew. Chem., Int. Ed. 2018, 57, 11952.

30. Billups, W. E.; Haley, M. M.; J. Am. Chem. Soc. 1991, 113, 5084.

31. Giannozzi, P.; Baroni, S.; Bonini, N.; Calandra, M.; Car, R.; Cavazzoni, C.; Ceresoli, D.; Chiarotti, G. L.; Cococcioni, M.; Dabo, I.; Dal Corso, A.; de Gironcoli, S.; Fabris, S.; Fratesi, G.; Gebauer, R.; Gerstmann, U.; Gougoussis, C.; Kokalj, A.; Lazzeri, M.; Martin-Samos, L.; Marzari, N.; Mauri, F.; Mazzarello, R.; Paolini, S.; Pasquarello, A.; Paulatto, L.; Sbraccia, C.; Scandolo, S.; Sclauzero, G.; Seitsonen, A. P.; Smogunov, A.; Umari, P.; Wentzcovitch, R. M.; J. Phys.: Condens. Matter 2009, 21, 395502.

32. Giannozzi, P.; Andreussi, O.; Brumme, T.; Bunau, O.; Buongiorno Nardelli, M.; Calandra, M.; Car, R.; Cavazzoni, 
C.; Ceresoli, D.; Cococcioni, M.; Colonna, N.; Carnimeo, I.; Dal Corso, A.; de Gironcoli, S.; Delugas, P.; DiStasio, R. A.; Ferretti, A.; Floris, A.; Fratesi, G.; Fugallo, G.; Gebauer, R.; Gerstmann, U.; Giustino, F.; Gorni, T.; Jia, J.; Kawamura, M.; Ko, H.-Y.; Kokalj, A.; Küçükbenli, E.; Lazzeri, M.; Marsili, M.; Marzari, N.; Mauri, F.; Nguyen, N. L.; Nguyen, H.-V.; Oterode-la-Roza, A.; Paulatto, L.; Poncé, S.; Rocca, D.; Sabatini, R.; Santra, B.; Schlipf, M.; Seitsonen, A. P.; Smogunov, A.; Timrov, I.; Thonhauser, T.; Umari, P.; Vast, N.; Wu, X.; Baroni, S.; J. Phys.: Condens. Matter 2017, 29, 465901.

33. Perdew, J. P.; Burke, K.; Ernzerhof, M.; Phys. Rev. Lett. 1996, 77, 3865.

34. Vanderbilt, D.; Phys. Rev. B 1990, 41, 7892.

35. Monkhorst, H. J.; Pack, J. D.; Phys. Rev. B 1976, 13, 5188.

36. Grimme, S.; Antony, J.; Ehrlich, S.; Krieg, H.; J. Chem. Phys. 2010, 132, 154104.

37. Pfrommer, B. G.; Côté, M.; Louie, S. G.; Cohen, M. L.; J. Comput. Phys. 1997, 131, 233.

38. Blöchl, P. E.; Jepsen, O.; Andersen, O. K.; Phys. Rev. B 1994, 49, 16223.

39. Baroni, S.; de Gironcoli, S.; Dal Corso, A.; Giannozzi, P.; Rev. Mod. Phys. 2001, 73, 515.

40. Gaillac, R.; Pullumbi, P.; Coudert, F.-X.; J. Phys.: Condens. Matter 2016, 28, 275201.
41. Chen, X.-Q.; Niu, H.; Li, D.; Li, Y.; Intermetallics 2011, 19, 1275.

42. VandeVondele, J.; Krack, M.; Mohamed, F.; Parrinello, M.; Chassaing, T.; Hutter, J.; Comput. Phys. Commun. 2005, 167, 103.

43. Hutter, J.; Iannuzzi, M.; Schiffmann, F.; VandeVondele, J.; Wiley Interdiscip. Rev.: Comput. Mol. Sci. 2014, 4, 15.

44. Goedecker, S.; Teter, M.; Hutter, J.; Phys. Rev. B 1996, 54, 1703.

45. Hartwigsen, C.; Goedecker, S.; Hutter, J.; Phys. Rev. B 1998, 58,3641 .

46. Ceriotti, M.; Bussi, G.; Parrinello, M.; Phys. Rev. Lett. 2009 , 102, 020601.

47. Zavitsas, A. A.; J. Phys. Chem. A 2003, 107, 897.

48. Lide, D. R.; Tetrahedron 1962, 17, 125.

49. Bradley, C. J.; Cracknell, A. P.; The Mathematical Theory of Symmetry in Solids: Representation Theory for Point Groups and Space Groups; OUP Oxford: New York, 2009.

50. Hehre, W. J.; Ditchfield, R.; Radom, L.; Pople, J. A.; J. Am. Chem. Soc. 1970, 92, 4796.

51. Mouhat, F.; Coudert, F.-X.; Phys. Rev. B 2014, 90, 224104.

52. Andrievski, R. A.; Int. J. Refract. Met. Hard Mater. 2001, 19 , 447. 